\title{
PROCESSAMENTO FONOLÓGICO E DESEMPENHO ESCOLAR NAS SÉRIES INICIAIS DO ENSINO FUNDAMENTAL
}

\author{
Phonological processing and school performance \\ in early grades of elementary school
}

\author{
Sabrina Ma Pimentel da Cunha Pinto Tenório ${ }^{(1)}$, Clara Regina Brandão de Ávila ${ }^{(2)}$
}

\begin{abstract}
RESUMO
Objetivo: caracterizar o desempenho escolar e o processamento fonológico de escolares da $1^{\underline{a}}$ e $2^{\underline{a}}$ séries, segundo o sexo e grau de escolaridade e investigar a existência de correlações entre essas variáveis. Método: participaram 88 escolares (48 meninos e 40 meninas) entre cinco e oito anos de idade, sem queixas relacionadas à fala ou à aprendizagem. Foram avaliados por meio do Teste de Desempenho Escolar (Stein,1994) e pelas provas de nomeação rápida, repetição de pseudopalavras e consciência fonológica, habilidades relacionadas ao processamento fonológico. A análise estatística se deu pelo teste de Mann-Withney $U$ e pelo coeficiente de Spearman, com intervalo de confiança de 95\%. Resultados: a maioria dos escolares apresentou classificação de desempenho inferior nos subtestes do Teste de Desempenho Escolar, com maior média de acertos para leitura. Não houve diferença significante entre meninos e meninas. Os escolares da $2^{\underline{a}}$ série mostraram melhor desempenho em escrita, leitura e aritmética, quando comparados aos alunos da $1^{\text {a }}$ série. Ao contrário dos desempenhos em leitura, escrita, aritmética e consciência fonológica, os desempenhos em acesso lexical e memória fonológica não diferenciaram as séries. Na 1aㅡ série, encontraram-se correlações positivas entre o acesso ao léxico mental e a consciência fonológica, entre leitura e escrita e consciência fonológica, e entre aritmética e consciência fonológica. $\mathrm{Na} 2^{\underline{a}}$ série, identificaram-se correlações positivas entre escrita, leitura e aritmética e com a consciência fonológica, acesso ao léxico com memória fonológica e consciência fonológica. Conclusão: os escolares da $2^{a}$ série apresentaram melhores escores de desempenho escolar e de consciência fonológica quando comparados aos alunos $1^{a}$, apesar da maioria ter alcançado classificação inferior à esperada para a série, definida pelo Teste. Diferentes variáveis do processamento fonológico correlacionaram-se positivamente com o desempenho escolar.
\end{abstract}

DESCRITORES: Leitura; Escrita Manual; Avaliação; Fonoaudiologia

\section{INTRODUÇÃO}

Há inúmeras evidências de que as habilidades de processamento fonológico são críticas para

(1) Fonoaudióloga; Professora do Departamento de Fonoaudiologia da Universidade Estadual de Alagoas; Especialização em Linguagem pela Universidade Federal de Pernambuco - UFPE; Mestranda do Programa de PósGraduação em Distúrbios da Comunicação Humana: Campo Fonoaudiológico da Universidade Federal de São Paulo - UNIFESP.

(2) Fonoaudióloga; Professora Associada do Departamento de Fonoaudiologia da Universidade Federal de São Paulo; Coordenadora do Núcleo de Ensino, Assistência e Pesquisa em Escrita e Leitura (NEAPEL) do Departamento de Fonoaudiologia - Universidade Federal de São Paulo.

Conflito de interesses: inexistente o aprendizado da leitura e da escrita, visto que aprender a ler por meio de um sistema alfabético pressupõe, dentre outras, a capacidade explícita de analisar a estrutura sonora da fala, bem como a de memória fonológica que permite reter informações e obter acesso a representações das informações fonológicas da linguagem ${ }^{1-3}$. A identificação e manipulação de segmentos articulados na fala encadeada são alcançadas a partir de atividades metacognitivas realizadas com informações fonológicas armazenadas na memória de longo prazo que possibilitam, também, a representação desses sons por meio da escrita. Os processos ligados à recepção (leitura) e expressão (escrita) mediados pela escrita são apreendidos por meio de atividades de decodificação e de codificação que vão desde 
a associação fonema-grafema até o conhecimento e uso de regras pré-estabelecidas que organizam a complexidade ortográfica. Geralmente, quando o aprendiz dá sinais de que pode identificar os segmentos da fala encadeada, ou seja, quando realiza tarefas de consciência fonológica, mostra que a associação fonema-grafema poderá suceder sem atropelos ou dificuldades.

De fato, certas pesquisas afirmaram que a estimulação da consciência fonológica facilita o subsequente aprendizado da leitura e da escrita ${ }^{4-7}$. Outras mostraram que a estimulação de habilidades metafonológicas, principalmente, a habilidade da consciência fonológica pode abreviar o aprendizado do princípio alfabético ou, ainda, remediar o aprendizado, quando alterações e dificuldades estão presentes ${ }^{8,9}$.

Memória operacional fonológica e consciência fonológica são habilidades complexas e estão interrelacionadas. São ambas, atividades constituintes do processamento fonológico. Algumas tarefas de consciência fonológica utilizadas igualmente na avaliação de capacidades preditoras do sucesso do aprendizado exigem e se utilizam de processos simples de recordação, enquanto outras exigem processos mais complexos de reconhecimento, envolvendo análise, retenção e comparação dos segmentos de fala encadeada ${ }^{10-12}$. Déficits de consciência fonológica podem prejudicar o aprendizado da leitura e da escrita em crianças. Essas dificuldades podem interferir no desempenho escolar ${ }^{13}$.

Apesar de aceita a relação entre as habilidades de processamento fonológico e o aprendizado da escrita alfabética, a direção da influência de uma habilidade sobre a outra, nessa relação, ainda não é consenso entre os pesquisadores. Alguns admitem que habilidades de consciência fonológica são um pré-requisito para o aprendizado da escrita e outros consideram a possibilidade da competência em leitura e escrita promover o desenvolvimento dos níveis mais refinados de processamento fonológico, demonstrando relação de causalidade recíproca entre essas habilidades ${ }^{14}$. Devido às características dessas habilidades básicas para o aprendizado do sistema alfabético, as crianças podem mostrar, no início dos anos escolares, diferenças de desempenho tanto para ler quanto para escrever, associadas a variações de competências no processamento das informações fonológicas. Essas variações podem ser observadas em tarefas ou atividades que avaliem o acesso ao léxico mental, a memória fonológica de curto prazo e a consciência fonológica. Segundo a literatura, essas diferenças ou variações podem ser mais facilmente percebidas nas séries iniciais ou na presença de transtornos do aprendizado ${ }^{15}$. A existência de correlação entre processamento fonológico e o desempenho escolar deve ser, portanto, observada nas séries iniciais do ensino fundamental.

Apesar da esperada diferença entre as séries escolares, não se observa um consenso entre as pesquisas sobre o desempenho de meninos e meninas em tarefas de processamento da informação fonológica. Por outro lado, o desempenho de meninas e meninas quanto ao desempenho escolar não mostra diferença quando analisados entre si6.

O objetivo desta pesquisa foi caracterizar o desempenho escolar e sua relação com o processamento fonológico em escolares de $1^{\underline{a}}$ e $2^{\underline{a}}$ séries, do ensino fundamental, segundo as variáveis sexo e ano escolar.

\section{MÉTODO}

Participaram desta pesquisa de correlação transversal, todos os escolares de $1^{\text {a }}$ e $2^{\underline{a}}$ séries, totalizando 88 , matriculados em uma escola pública da rede municipal de ensino, segundo os critérios de inclusão. Os sujeitos tinham entre cinco e oito anos de idade, e foram distribuídos da seguinte forma: 43 escolares da $1^{\text {a }}$ série $(23$ meninos e 20 meninas) e 45 escolares da $2^{\text {a }}$ série $(25$ meninos e 20 meninas). Foram selecionados segundo os critérios de inclusão definidos a priori: estarem matriculados na $1^{\text {a }}$ ou na $2^{\text {a }}$ série escolar; ausência de queixas ou indícios, declarados por seus professores, de déficits sensoriais e/ou motores, de distúrbios cognitivos e/ou neurológicos, de transtornos psico-afetivos ou de transtornos ou dificuldades de aprendizagem; frequência mínima de $75 \%$ às aulas. Todos os 88 escolares cumpriram os critérios de inclusão e foram avaliados.

Os escolares foram avaliados, individualmente, em sessão que durou, em média, 50 minutos. Todas as avaliações seguiram um protocolo único, e a mesma ordem de aplicação das 06 tarefas de avaliação: do desempenho escolar em tarefas de escrita, aritmética e leitura, e do processamento fonológico em provas de avaliação do acesso ao léxico mental, da memória fonológica de trabalho e da consciência fonológica.

A avaliação do desempenho escolar foi realizada por meio da aplicação do Teste de Desempenho Escolar - TDE ${ }^{16}$, constituído pelos subtestes de: escrita, com 34 palavras ditadas pela examinadora e a escrita do próprio nome do escolar; aritmética com três perguntas envolvendo solução de problemas e 35 cálculos matemáticos; e leitura com 70 palavras isoladas a serem lidas em voz alta pelo escolar. Os escores brutos de cada subteste foram calculados pelo número de acertos e o seu somatório representou o escore bruto total (EBT), 
correspondendo ao desempenho escolar do aluno, classificado segundo a gradação superior, médio ou inferior, conforme instruções do próprio TDE.

O processamento fonológico (PF) foi avaliado por meio da aplicação de três diferentes instrumentos: o Brazilian Children's Test of Pseudoword Repetition - $\mathrm{BCPR}^{17}$, para avaliar a memória fonológica de trabalho, composto por 40 pseudopalavras, distribuídas segundo as relações de baixa, média e alta similaridade com palavras reais; a prova de nomeação automatizada rápida - NRA - subteste objetos - ${ }^{18}$ para avaliar o acesso ao léxico mental, composta por 36 figuras de 06 objetos, distribuídas em quatro linhas e nove colunas, (figuras apresentadas inicialmente, para reconhecimento do escolar; e, a prova de consciência fonológica PCF ${ }^{8}$, constituída por dez subtestes, com quatro itens cada, para avaliar habilidades de manipulação de fonemas e sílabas.

As médias de escore bruto de cada subteste do TDE e as médias de acerto de cada uma das avaliações do processamento fonológico foram calculadas para cada série, e comparadas e correlacionadas, por subteste ou prova e pelo ano escolar. Calcularam-se, também as médias de Escore Bruto Total do TDE, por série, assim como a média dos totais de acertos em todas as avaliações do Processamento Fonológico.

Esta pesquisa foi iniciada após a aprovação pelos Comitês de Ética e Pesquisa da UNIFESP $\left(n^{\circ}\right.$ de protocolo: 0543/09) e da UNCISAL ( $n^{\circ}$ de protocolo: 744/07). Todos os responsáveis pelos escolares assinaram o Termo de Consentimento Livre e Esclarecido após terem recebido informações sobre os procedimentos realizados e os escolares assentiram sua participação na pesquisa.

Os resultados foram analisados estatisticamente por meio do teste de Mann-Whitney U para comparação das variáveis desempenho escolar e processamento fonológico, e o teste de correlação bivariada por meio do Coeficiente de Spearman, para verificar a correlação entre essas variáveis, tanto na $1^{\underline{a}}$ quanto na $2^{\underline{a}}$ série. Os valores de correlação foram considerados segundo a classificação de Maxwell e Satake (1997): Icl < 0,40 para correlação fraca, $0,40<$ Icl $<0,70$ para correlação moderada, $0,70<\mathrm{Icl}<0,90$ para correlação boa, e Icl $>0,90$ para correlação ótima. Para a análise estatística utilizou-se o programa SPSS (Statistical Package for Social Sciences), considerando os valores significantes para p-valor menor que $0,05(p<0,05)$, sendo de $95 \%$ o intervalo de confiança admitido.

\section{RESULTADOS}

A comparação entre as médias de acerto alcançadas por meninos e meninas, em cada subteste do TDE e de PF não mostrou diferença estatisticamente significante entre os sexos, em nenhuma das séries. Meninos e meninas tiveram desempenhos semelhantes independentemente da série escolar que freqüentavam (Tabela1).

Tabela 1 - Distribuição das médias de acertos dos escolares da $1^{\mathrm{a}}$ e $2^{\mathrm{a}}$ séries, segundo o sexo e os subtestes do TDE e os testes de PF

\begin{tabular}{|c|c|c|c|c|c|c|c|c|c|c|c|}
\hline \multirow{2}{*}{\multicolumn{2}{|c|}{ Subtestes e Provas }} & \multicolumn{5}{|c|}{$1^{\text {a }}$ Série } & \multicolumn{5}{|c|}{$2^{a}$ Série } \\
\hline & & \multicolumn{2}{|c|}{ Média } & \multicolumn{2}{|c|}{ DP } & \multirow{2}{*}{ p-valor } & \multicolumn{2}{|c|}{ Média } & \multicolumn{2}{|c|}{ DP } & \multirow{2}{*}{ p-valor } \\
\hline & & M & $\mathbf{F}$ & M & $\mathbf{F}$ & & $\mathbf{M}$ & $F$ & M & $\mathbf{F}$ & \\
\hline \multirow{4}{*}{ TDE } & Escrita & 4,0 & 2,7 & 4,0 & 4,3 & 0,164 & 8,7 & 8,2 & 5,9 & 6,6 & 0,748 \\
\hline & Aritmética & 2,0 & 1,7 & 2,7 & 2,4 & 0,615 & 6,7 & 5,7 & 2,8 & 2,9 & 0,282 \\
\hline & Leitura & 14,9 & 15,6 & 23,5 & 26,7 & 0,526 & 47,3 & 41,3 & 20,9 & 23,6 & 0,602 \\
\hline & EBT & 20,9 & 20,0 & 21,7 & 30,7 & 0,401 & 62,8 & 55,2 & 27,3 & 30,2 & 0,530 \\
\hline \multirow{4}{*}{ PF } & NRA & 30,6 & 31,4 & 3,5 & 2,6 & 0,555 & 31,0 & 30,9 & 3,2 & 2,5 & 0,598 \\
\hline & BCPR & 36,8 & 37,4 & 2,7 & 3,2 & 0,254 & 38,1 & 37,9 & 2,0 & 2,4 & 0,678 \\
\hline & PCF & 14,2 & 16,9 & 6,2 & 6,8 & 0,360 & 22,9 & 21,7 & 6,8 & 6,3 & 0,713 \\
\hline & PF & 81,6 & 85,7 & 21,9 & 22,0 & 0,252 & 92,0 & 90,5 & 22,4 & 22,0 & 0,545 \\
\hline
\end{tabular}

Legenda: STDE= Subtestes do Teste de Desempenho Escolar; EBT= Escore Bruto Total; PF= Processamento Fonológico; PFT= Processamento Fonológico Total; DP= Desvio Padrão, $M=$ Masculino, $F=$ Feminino, NRA= Nomeação Rápida - parte $A$, BCPR= Repetição de Pseudopalavras, PCF= Prova de Consciência Fonológica. Teste Mann-Whitney U. Valor de $p<0,05$ 
Os escolares da 2a série mostraram maiores médias de acertos em todos os subtestes do TDE, além de melhores respostas nas tarefas de PCF e $\mathrm{PF}$ total, quando comparados aos escolares da $1^{\text {a }}$ série $(p=0,000)$. No entanto, os desempenhos nas tarefas de NRA e de BCPR foram semelhantes quando comparados os anos escolares, sendo as únicas habilidades, dentre as pesquisadas, que não apresentaram diferença estatisticamente significantes (Tabela 2).

Tabela 2 - Médias de escores e de acertos e desvios-padrão dos escolares da 1⿳亠丷厂 e 2a séries, independentemente do sexo, distribuídas segundo os subtestes do TDE e os testes de Processamento Fonológico

\begin{tabular}{ccccccc}
\hline & & \multicolumn{2}{c}{ 1 $^{\text {a Série }}$} & \multicolumn{2}{c}{ 2 $^{\text {a Série }}$} & \multirow{2}{*}{ p-valor } \\
\cline { 3 - 6 } & & Média & DP & Média & DP & \\
\hline \multirow{4}{*}{ STDE } & Escrita & 3,3 & 4,2 & 8,6 & 6,2 & $0,00^{*}$ \\
& Aritmética & 1,8 & 2,6 & 6,3 & 2,9 & $0,00^{*}$ \\
& Leitura & 15,6 & 25,0 & 45,6 & 21,3 & $0,00^{*}$ \\
& EBT & 20,9 & 28,8 & 60,6 & 27,6 & $0,00^{*}$ \\
\hline \multirow{4}{*}{ PF } & NRA & 31,0 & 3,1 & 31,0 & 2,9 & 0,823 \\
& BCPR & 37,0 & 2,9 & 38,0 & 2,2 & 0,197 \\
& PCF & 15,4 & 6,6 & 22,4 & 6,4 & $0,00^{*}$ \\
& PF & 83,4 & 22,2 & 91,4 & 21,7 & $0,000^{*}$ \\
\hline
\end{tabular}

Legenda: STDE= Subtestes do Teste de Desempenho Escolar, EBT= Escore Bruto Total, PF= Processamento Fonológico, NRA= Nomeação Rápida - parte A, BCPR= Repetição de Pseudopalavras, PCF= Prova de Consciência Fonológica, DP= Desvio Padrão. Teste de Mann-Withney. Valor de $p<0,05$

Um maior número de escolares da $2^{\text {a }}$ série mostrou desempenho classificado como inferior, no TDE, quando comparadas as séries nos subtestes de escrita e aritmética. As diferenças encontradas entre as duas séries mostraram-se estatisticamente significantes. No subteste de leitura, os escolares de ambas as séries obtiveram desempenho semeIhante quanto às classificações do TDE (Tabela 3).

Tabela 3 - Distribuição dos escolares da $1^{\mathrm{a}}$ e $2^{\mathrm{a}}$ séries, segundo as classificações nos subtestes do TDE

\begin{tabular}{|c|c|c|c|c|c|c|c|c|}
\hline \multirow{3}{*}{$\begin{array}{l}\text { Subtestes } \\
\text { do TDE }\end{array}$} & \multirow{3}{*}{ Série } & \multicolumn{6}{|c|}{ Classificações do TDE } & \multirow{3}{*}{ p-valor } \\
\hline & & \multicolumn{2}{|c|}{ Superior } & \multicolumn{2}{|c|}{ Médio } & \multicolumn{2}{|c|}{ Inferior } & \\
\hline & & $\mathbf{N}$ & $\%$ & $\mathbf{N}$ & $\%$ & $\mathbf{N}$ & $\%$ & \\
\hline \multirow{2}{*}{ Escrita } & $1^{\mathrm{a}}$ & 0 & 0 & 17 & 39,5 & 26 & 60,4 & \multirow{2}{*}{$0,00^{*}$} \\
\hline & $2^{\mathrm{a}}$ & 0 & 0 & 3 & 6,6 & 42 & 93,3 & \\
\hline \multirow{2}{*}{ Aritmética } & $1^{a}$ & 1 & 2,3 & 14 & 32,5 & 28 & 65,1 & \multirow{2}{*}{$0,014^{*}$} \\
\hline & $2^{\mathrm{a}}$ & 0 & 0 & 5 & 11,1 & 40 & 88,8 & \\
\hline \multirow{2}{*}{ Leitura } & $1^{a}$ & 3 & 6,9 & 13 & 30,2 & 27 & 62,7 & \multirow{2}{*}{0,514} \\
\hline & $2^{\mathbf{a}}$ & 6 & 13,3 & 13 & 28,8 & 26 & 57,7 & \\
\hline \multirow{2}{*}{ EBT } & $1^{a}$ & 0 & 0 & 17 & 39,5 & 26 & 60,4 & \multirow{2}{*}{$0,024^{*}$} \\
\hline & $2^{\mathrm{a}}$ & 0 & 0 & 8 & 17,7 & 37 & 82,2 & \\
\hline
\end{tabular}

Legenda: TDE $=$ Teste de Desempenho Escolar, EBT= Escore bruto total, $\mathrm{N}=$ número absoluto dos escolares, \%= percentual dos escolares. Teste de Mann-Withney. Valor de $p<0,05$

Conforme os dados observados na Tabela 4, identificou-se correlação positiva moderada entre o desempenho escolar e o processamento fonológico, quando foram analisados os resultados da $1^{\text {a }}$ série. Observou-se também correlações positivas ótima entre o processamento fonológico e a prova de consciência fonológica, moderada entre o desempenho em escrita e o observado em leitura, fraca entre nomeação rápida e a escrita, fraca entre repetição de pseudopalavras e aritmética, 
moderada entre a prova de consciência fonológica e a escrita e correlação positiva boa entre a prova de consciência fonológica e a leitura. Observaramse, ainda, correlações positivas moderada entre a nomeação rápida e a consciência fonológica, fraca entre repetição de pseudopalavras e prova de consciência fonológica, e moderada entre desempenho em escrita e desempenho em leitura, nas análises da $1^{\text {a }}$ série.

Tabela 4 - Correlações entre os desempenhos nos subtestes do TDE, e seu escore bruto total, e os testes do PF nos escolares da $1^{\text {a }}$ série, independentemente do sexo

\begin{tabular}{|c|c|c|c|c|c|c|c|c|c|c|c|c|c|c|}
\hline \multirow{2}{*}{ TPF/STDE } & \multicolumn{2}{|c|}{ DE } & \multicolumn{2}{|c|}{ DA } & \multicolumn{2}{|c|}{ DL } & \multicolumn{2}{|c|}{ NRA } & \multicolumn{2}{|c|}{ BCPR } & \multicolumn{2}{|c|}{ PCF } & \multicolumn{2}{|c|}{ PFT } \\
\hline & C & p-valor & C & p-valor & C & p-valor & C & p-valor & C & p-valor & C & p-valor & C & p-valor \\
\hline NRA & ---- & ---- & ----- & ---- & ----- & ------ & 1,0 & --- & 0,199 & 0,200 & 0,516 & $0,00^{*}$ & 0,707 & $0,00^{*}$ \\
\hline BCPR & ----- & ----- & ------ & ----- & ----- & ------ & 0,199 & 0,200 & 1,0 & --- & 0,381 & $0,012^{*}$ & 0,556 & $0,004^{*}$ \\
\hline PCF & ---- & ----- & ------ & ----- & ----- & ------ & 0,516 & $0,00^{*}$ & 0,381 & $0,012^{*}$ & 1,0 & --- & 0,922 & $0,00^{*}$ \\
\hline DE & 1,0 & ---- & 0,203 & 0,192 & 0,652 & $0,00^{*}$ & 0,357 & $0,019^{*}$ & 0,077 & 0,622 & 0,663 & $0,00^{*}$ & 0,605 & $0,000^{*}$ \\
\hline DA & -- & ---- & ---- & --- & 0,108 & 0,491 & 0,195 & 0,209 & $-0,377$ & $0,013^{*}$ & 0,039 & 0,805 & 0,000 & 0,997 \\
\hline DL & ------ & ----- & ---- & -------- & 1,0 & --- & 0,257 & 0,096 & 0,297 & 0,053 & 0,734 & $0,00^{*}$ & 0,667 & $0,001^{*}$ \\
\hline EBT & 0,661 & $0,00^{*}$ & 0,442 & $0,003^{*}$ & 0,882 & $0,00^{*}$ & 0,251 & 0,104 & 0,035 & 0,822 & 0,664 & $0,00^{*}$ & 0,661 & $0,00^{*}$ \\
\hline
\end{tabular}

Legenda: TPF= Testes de Processamento Fonológico, NRA= Nomeação Rápida - Parte A, BCPR= Repetição de Pseudopalavras, $\mathrm{PCF}=$ Prova de Consciência Fonológica, PFT= Processamento Fonológico Total, STDE= Subtestes do Teste de Desempenho Escolar, $\mathrm{DE}=$ Desempenho em Escrita, $\mathrm{DA}=$ Desempenho em Aritmética, DL= Desempenho em Leitura, EBT= Escore Bruto Total, $\mathrm{c}=$ correlação. Teste de Correlação Bivariada - Coeficiente de Spearman.

Da mesma forma, os resultados da Tabela 5 mostram a presença de correlações positivas moderada na $2^{\text {a }}$ série do desempenho escolar com o processamento fonológico e a consciência fonológica, e ótima do processamento fonológico e consciência fonológica. Houve ainda correlações positivas moderada do processamento fonológico e da consciência fonológica com todos os subtestes do teste de desempenho escolar. Além disso, foram evidenciadas correlações positivas moderada entre a nomeação rápida e a repetição de pseudopalavras, e fraca com a consciência fonológica; correlações positivas moderada entre o desempenho em escrita e o desempenho em aritmética, boa entre os desempenhos em escrita e em leitura, e moderada entre desempenho em aritmética e desempenho em leitura.

Tabela 5 - Correlações entre os desempenhos nos subtestes do TDE, e seu escore bruto total e os testes do PF nos escolares da $2^{\mathrm{a}}$ série, independentemente do sexo

\begin{tabular}{|c|c|c|c|c|c|c|c|c|c|c|c|c|c|c|}
\hline \multirow{2}{*}{ PF/STDE } & \multicolumn{2}{|c|}{ DE } & \multicolumn{2}{|c|}{ DA } & \multicolumn{2}{|c|}{ DL } & \multicolumn{2}{|c|}{ NRA } & \multicolumn{2}{|c|}{ BCPR } & \multicolumn{2}{|c|}{ PCF } & \multicolumn{2}{|c|}{ PFT } \\
\hline & C & p-valor & C & p-valor & C & p-valor & C & p-valor & C & p-valor & C & p-valor & C & p-valor \\
\hline NRA & ----- & ----- & ------- & ----- & ----- & ------ & 1,0 & --- & 0,615 & $0,00^{*}$ & 0,326 & $0,031^{*}$ & 0,613 & $0,00^{*}$ \\
\hline BCPR & ----- & ----- & ------ & ----- & ----- & ------ & 0,615 & $0,00^{*}$ & 1,0 & --- & 0,258 & 0,091 & 0,538 & $0,00^{*}$ \\
\hline PCF & ----- & --.-- & ------- & ----- & ----- & ------- & 0,326 & $0,031^{*}$ & 0,258 & 0,091 & 1,0 & --- & 0,913 & $0,00^{*}$ \\
\hline $\mathrm{DE}$ & 1,0 & -- & 0,611 & $0,00^{*}$ & 0,760 & $0,00^{*}$ & 0,157 & 0,309 & 0,190 & 0,217 & 0,619 & $0,00^{*}$ & 0,537 & $0,000^{*}$ \\
\hline DA & ---- & ---- & 1,0 & --- & 0,454 & $0,002^{*}$ & 0,250 & 0,102 & 0,112 & 0,469 & 0,609 & $0,00^{*}$ & 0,555 & $0,00^{*}$ \\
\hline DL & ------ & ------ & 0,454 & $0,002^{*}$ & 1,0 & --- & 0,052 & 0,737 & 0,120 & 0,439 & 0,522 & $0,00^{*}$ & 0,450 & $0,002^{*}$ \\
\hline EBT & 0,891 & $0,00^{*}$ & 0,582 & $0,00^{*}$ & 0,955 & $0,00^{*}$ & 0,083 & 0,593 & 0,123 & 0,428 & 0,579 & $0,00^{*}$ & 0,550 & $0,007^{*}$ \\
\hline
\end{tabular}

Legenda: STDE= Subtestes do Teste de Desempenho Escolar, DE= Desempenho em escrita, $\mathrm{DA}=$ Desempenho em Aritmética, $\mathrm{DL}=$ Desempenho em Leitura, EBT= Escore bruto total, NRA= Nomeação Rápida - Parte A, BCPR= Repetição de Pseudopalavras, PCF= Prova de Consciência Fonológica, PFT= Processamento Fonológico, c=correlação. Teste de Correlação Bivariada - Coeficiente de Spearman. 


\section{DISCUSSÃO}

Nesta pesquisa, meninos e meninas da mesma série apresentaram desempenhos semelhantes nas atividades avaliadas sugerindo não haver influência do sexo no desempenho escolar em escrita, leitura e aritmética e nas habilidades do processamento fonológico, nas séries iniciais do EF. Outros estudos encontraram resultados semelhantes quando pesquisaram o desenvolvimento da escrita em grupos de meninos e meninas da mesma, na faixa etária ${ }^{19-21}$.

A partir desse resultado, meninos e meninas foram reunidos e cada série estudada como um grupo. As médias de acertos, ou seja, das médias de escores encontradas para cada variável pesquisada foram comparadas entre as séries (Tabela 2). Os resultados mostraram que os escolares da $2^{\text {a }}$ série apresentaram maior número de acertos nas provas de escrita, leitura e aritmética quando comparados aos da $1^{\text {a }}$ série. Uma vez que o TDE apresenta tarefas e estímulos únicos para todas as séries que avalia, esse resultado já era esperado. Por outro lado, à Tabela 3 , pôde-se perceber que quando classificados pelo escore bruto, segundo os parâmetros de classificação do desempenho do TDE, um maior número de escolares da $2^{\mathrm{a}}$ série obtiveram pior classificação de desempenho que os alunos de $1^{\underline{a}}$ série, embora tenham tido melhores resultados numéricos no Teste. Estes dados ressaltam que as exigências dos parâmetros para a classificação proposta pelo TDE aumentam, segundo o nível de escolaridade, o que certamente, influenciou a obtenção de pior classificação da $2^{\text {a }}$ série quando comparada à 1 a

Dentre as habilidades de processamento fonológico, a consciência fonológica e a média geral do processamento fonológico, puderam diferenciar as séries evidenciando melhor desempenho da $2^{2}{ }^{a}$ série (Tabela 2). Esse resultado mostra, como esperado, que a progressão da idade e, principalmente, dos anos escolares, influencia o desenvolvimento e o aperfeiçoamento das habilidades de consciência fonológica que dependem, em parte, do contato com a escrita e do aprendizado. De fato, a literatura indica a presença de correlação com o tempo e o nível de escolaridade ${ }^{7,22}$. Contrariamente, as demais habilidades de acesso ao léxico mental e memória fonológica de trabalho, desenvolvidas ao longo do crescimento e importantes tanto para o desenvolvimento da comunicação oral quanto para o aprendizado da escrita ${ }^{23}$, mostraram-se semelhantes em ambas as séries pesquisadas.

Os escores mais altos de consciência fonológica dos escolares da $2^{2}$ série mostram a força da relação entre a consciência fonológica, a leitura e a escrita ${ }^{3,24}$, uma vez que nessa série, os escores de leitura e de escrita, também se mostraram melhores que os valores da $1^{\text {a }}$ série. Não houve diferença entre as séries quanto às habilidades de memória fonológica de curto prazo e acesso ao léxico mental, sugerindo que estas habilidades independeram da estimulação escolar ou da idade nessa amostra de escolares.

O estudo das relações entre as variáveis de desempenho escolar e de processamento fonológico estudadas na $1^{\text {a }}$ série (Tabela 4), evidenciou a presença de correlação entre o desempenho escolar e o processamento fonológico, e entre o escore bruto total do desempenho escolar e a consciência fonológica, mostrando que quanto mais acertos nas tarefas que envolveram as habilidades metafonológicas de consciência fonológica, melhor o desempenho escolar geral.

A literatura indica que ler e escrever são capacidades fortemente relacionadas às habilidades de consciência fonológica, principalmente nos estágios iniciais do aprendizado, ou seja, nas primeiras séries escolares ${ }^{1,15}$. A consciência fonológica desempenha um papel importante na aquisição da leitura em um sistema de escrita alfabético. De uma forma geral, as letras do alfabeto representam fonemas tornando fácil compreender que a habilidade de identificar, segmentar e manipular fonemas se correlacione com o aprendizado do princípio alfabético da escrita, base para a leitura e a escrita ${ }^{25}$. A correlação entre acesso ao léxico e o desempenho em escrita, evidenciada no grupo de escolares da $1^{\text {a }}$ série, segundo os dados da Tabela 4 confirma a idéia de existência de relação entre a habilidade de reconhecer palavras escritas, rápida e sucessivamente, e a habilidade de reconhecimento rápido de estímulos visuais que exige o uso de recursos do executivo central, como atenção, discriminação e memória de curto e longo prazo $0^{9,26-28}$. A melhor leitura concorreria para facilitar o processamento ortográfico, cuja expressão se mostraria na escrita correta de palavras. A correlação da consciência fonológica tanto com o desempenho em escrita quanto com a leitura, nos escolares da $1^{\underline{a}}$ série, também foi relatada em diferentes pesquisas ${ }^{18,20,23}$. Para escrever, é necessário que a criança resgate as informações fonológicas adquiridas e, conscientemente, manipule os sons da fala, reconhecendoos para convertê-los em grafemas 3 ,25,28-38. Também foi encontrada correlação positiva entre o desempenho em aritmética e o desempenho em leitura, corroborando os achados de uma pesquisa prévia ${ }^{38}$.

As correlações verificadas nos resultados dos escolares da $2^{\text {a }}$ série, da CF e do processamento fonológico geral com os três desempenhos escolares avaliados e o desempenho escolar total, 
mostram que para escrever, nessa série, ainda são necessários os resgates das informações fonológicas e, dessa forma, corroboram pesquisas na área ${ }^{3,25,30-36,38,39}$.

A correlação do acesso ao léxico com a memória fonológica de trabalho era esperada, uma vez que só é possível acessar informações que estiverem armazenadas em um sistema íntegro de memória ${ }^{7,40}$. As correlações entre a escrita e a leitura podem evidenciar que mesmo na $2^{\mathrm{a}}$ série os escolares ainda utilizam com a mesma intensidade, as habilidades fundamentais ligadas à capacidade de associação fonema-grafema, tanto para ler quanto para escrever.

Os resultados podem mostrar que mesmo na $2^{\text {a }}$ série ler e escrever são tarefas fortemente dependentes do processamento fonológico da informação lingüística. Podem sugerir, também, que o domínio do princípio alfabético pode despender mais tempo assim como, em conseqüência o aprendizado da escrita e da leitura ortográficas.

Os achados desta pesquisa sinalizam a importância de investigar mais detalhadamente as relações do desempenho da criança em seu ambiente escolar. Sinaliza, também, as possibilidades de atuação junto ao sistema educacional, de modo estabelecer programas de estimulação do rápido acesso às informações fonológicas minimizando as dificuldades próprias do aprendizado da leitura e da escrita, favorecendo o desenvolvimento acadêmico e otimizando as potencialidades do alunado que terá mais oportunidades de avanço nesse processo. Novas pesquisas devem ser feitas para o esclarecimento sobre as habilidades de processamento fonológico nas séries escolares subseqüentes.

\section{CONCLUSÃO}

Não foram encontradas diferenças estatisticamente significantes entre os desempenhos de meninos e meninas. Como esperado, os escolares da $2^{\text {a }}$ série mostraram melhor desempenho em escrita, leitura e aritmética, apesar da indicação de maior número de escolares da $2^{\underline{a}}$ série com desempenho escolar inferior quando comparados aos da $1 \frac{a}{-}$ série. Ao contrário da leitura, escrita, aritmética e consciência fonológica, os desempenhos em acesso lexical e memória fonológica não diferenciaram as séries. Identificaram-se correlações positivas entre o desempenho escolar e o processamento fonológico em ambas as séries.

\begin{abstract}
Purpose: to characterize school performance and phonological processing of First and Second Grade students according to the following variables: gender; writing, arithmetic, and reading performance; lexical phonological access; phonological short-term memory; and phonological awareness, investigating the presence of correlations between groups and among variables. Method: eightyeight students (boys and girls) with ages between 05 years and 08 years, without speech or learning complaints, took part in the study. Participants had their performance assessed on: writing, reading and arithmetic tasks; subtests of the School Performance Test (Stein, 1994); lexical access; short term phonological memory; and skills related to phonological processing. Data were tabulated and analyzed through Mann-Whitney $U$ test for comparison of school performance variables and phonological processing. Spearman coefficient was used to check the correlation among such variables. The confidence interval adopted was $95 \%$. Results: most students showed lower performance on School Performance Test' subtests, with higher mean accuracy as for reading. No significant difference was observed between boys and girls. In the First Grade group, good to moderate positive correlations between lexical access and phonological awareness; reading and writing and phonological awareness; arithmetic and phonological awareness were observed. In the Second Grade group, good to moderate positive correlations among writing, reading and arithmetic; phonological awareness, lexical access, phonological memory and phonological awareness were observed. Conclusions: second grade students showed better performance on writing, reading and phonological awareness. In contrast to performance on writing, reading and arithmetic, performance on lexical access and phonological memory did not differ between grades. Good to moderate positive correlations between school performance and phonological processing were observed.
\end{abstract}

KEYWORDS: Reading; Handwriting; Evaluation; Speech, Language and Hearing Sciences 


\section{REFERÊNCIAS}

1. Torgesen JK, Wagner RK, Rashotte CA. Longitudinal studies of phonological processing and reading. Journal of Learning Disabilities, 1994, 27(5): 276-86.

2. Simões, VF. Estudo do desempenho de crianças das séries iniciais o ensino fundamental I em testes de leitura, escrita e nomeação rápida. [Dissertação], São Paulo: Universidade de São Paulo, 2006.

3. Mousinho R, Corre J. Linguistic and cognitive skills in readers and nonreaders. Pró-Fono Rev de Atualização Científica. 2009, abr-jun; 21(2): 113-8.

4. Lundberg I, Frost J, Petersen O. Effects of an extensive program for stimulating phonological awareness in preschool children. Reading Research Quarterly, 1988, 23: 262-84.

5. Allor, JH. The relationships of phonemic awareness and rapid naming to reading development. Learning Disability Quarterly. 2002; 25, 47-57.

6. Cardoso-Martins C, Pennington BF. The relationship between phoneme awareness and rapid naming skills and literacy acquisition: the role of development period and reading ability. Sci. Stud. Read. 2004; 8(1): 27-52.

7. Silva C, Capellini SA. Eficácia do programa de remediação fonológica e leitura no distúrbio de aprendizagem. Pró-Fono Revista de Atualização Científica. 2010, abr-jun; 22(2): 131-8.

8. Capovilla AGS, Capovilla FC. Prova de consciência fonológica: desenvolvimento de dez habilidades da pré-escola à segunda série. Temas sobre Desenvolvimento. 1998; 7(37): 14-35.

9. Salgado CA, Capellini SA. Programa de remediação fonológica em escolares com dislexia do desenvolvimento. Pró-Fono Rev Atual. Cient. 2008; 20(1): 31-6.

10. Ferreira TL, Capellini AS, Ciasca SM, Tonelotto JMF. Desempenho de escolares leitores proficientes no teste de nomeação automatizada rápida (RAN). Temas sobre Desenvolvimento. 2003; 12(69): 26-32.

11. Barbosa, T. Memória operacional fonológica, consciência fonológica e linguagem nas dificuldades de alfabetização [Tese]. São Paulo: Universidade Federal de São Paulo, 2005.

12. Nicolielo AP, Fernandes GB, Garcia VL, Hage SRV. Desempenho escolar de crianças com distúrbio específico de linguagem: relações com habilidades metafonológicas e memória de curto prazo. Rev. Soc Bras Fonoaudiol. 2008; 13(3): 246-50.

13. Santos MTM, Navas ANGP. Aquisição e desenvolvimento da linguagem escrita. In: Santos MTM, Navas ANGP. Distúrbios de leitura e escrita: teoria e prática. Ed. Manole, 2002, p. 1-26.
14. Gathercole SE, Alloway TP, Willis C, Adams AM. Working memory in children with reading disabilities. Journal of Experimental Child Psychology. 2006; 93: 265-81.

15. Avila CRB, Carvalho CAF, Batista AS. Parâmetros de fluência e compreensão de leitura. In: Barbos T, Rodrigues CC, Mello CB, Capellini SA, Mousinho R, Alves LM (org). Temas em Dislexia. São Paulo: Artes Médicas, 2009, p. 73-88.

16. Stein LM. TDE; teste de desempenho escolar: manual para aplicação e interpretação. São Paulo: Casa do Psicólogo, 1994.

17. Santos $\mathrm{FH}$, Bueno OFA. Validation of the Brazilian Children's Test of Pseudoword Repetition in Portuguese speakers aged 4 to 10 years. Brazilian Journal of Medical Biological Research. 2003; 36: 1533-47.

18. Rosal CAR. Habilidades de segmentação fonêmica em crianças normais de primeira, segunda e terceira séries do ensino fundamental. [Tese]. São Paulo: Universidade de São Paulo - USP, 2002.

19. Flores-Mendoza CE, Mansur-Alves M, Lelé AJ, Bandeira DR. Inexistência de diferenças de sexo no fator $\mathrm{g}$ (inteligência geral) e nas habilidades específicas em crianças de duas capitais brasileiras. Psicologia: reflexão e crítica. 2007; 20(3): 499-506. 20. Gindri, G. Memória de trabalho, consciência fonológica e hipótese de escrita: um estudo com alunos de pré-escola e de primeira série. [Mestrado] Santa Maria: Universidade Federal de Santa Maria, 2006.

21. Andreazza-Balestrin C, Cielo CA, Lazzarotto C. Relação entre desempenho em consciência fonológica e a variável sexo: um estudo com crianças pré-escolares. Rev. Soc Bras Fonoaudiol. 2008; 13(2): 154-60.

22. Salles JF, Parente MAMP. Funções neuropsicológicas em crianças com dificuldades de leitura e escrita. Psicologia: teoria e pesquisa. 2006; 22(2): 153-62.

23. Araújo MR, Minervino CASM. Avaliação cognitiva: leitura, escrita e habilidades relacionadas. Psicologia em Estudo.2008, out/dez; 13(4): 859-65. 24. Nunes C, Frota S, Mousinho R. Consciência fonológica e o processo de aprendizagem de leitura e escrita: implicações teóricas para o embasamento da prática fonoaudiológica. Rev. CEFAC. 2009, abr/ jun; 11(2): 207-12.

25. Cardoso-Martins C, Pennington BF. What is the contribution of rapid serial naming to reading and spelling ability?: evidence from children and adolescents with and without reading difficulties. Psicolologia: reflexão e crítica. 2001; 14(2): 387-97. 26. Baddeley AD. Working Memory and Language: an overview. Journal Communication Disorders. 2003; 36: 189-208. 
27. Bicalho LGR, Alves LM. A nomeação seriada rápida em escolares com e sem queixas de problemas de aprendizagem em escola pública e particular. Rev CEFAC. 2010; 12(4): 608-16.

28. Capellini SA, Conrado TLBC. Desempenho de escolares com e sem dificuldades de aprendizagem de ensino particular em habilidade fonológica, nomeação rápida, leitura e escrita. Rev. CEFAC. 2009;11(supl 2):183-93.

29. Mota HB, Filha MGCM, Lasch SS. A consciência fonológica e o desempenho na escrita sob ditado de crianças com desvio fonológico após realização de terapia fonoaudiológica. Rev CEFAC. 2007; 9(4): 477-82.

30. Mann, VA; Liberman, IY. Phonological awareness and verbal short-term memory. Journal of learning disabilities. Chicago. 1984; 17(10): 592-9.

31. Blischak DM. Phonological awareness: implications for individuals with little or no functional speech. Augmentative Alternative Commun. 1994; 10(4): 245-54.

32. Share D. Phonological recoding and sel-teaching: sine qua nono f reading acquisition. Cognition. 1995; 55:151-218.

33. Cárnio MS, Santos D. Evolução da consciência fonológica em alunos de ensino fundamental. Pró-Fono Rev. Atual. Cient. 2005; 17(2): 195-200.
34. Paes CTS, Pessoa ACRG. Habilidades fonológicas em crianças não alfabetizadas e alfabetizadas. Rev. CEFAC. 2005; 7(2): 149-57. 35. Pestun MSV. Consciência fonológica no início da escolarização e o desempenho ulterior em leitura e escrita: estudo correlacional. Estud. Psicol. 2005; 10(3): 407-12.

36. Tirapegui CJC, Gajardo LRC, Ortiz ZB. Conciencia fonológica y lengua escrita em niños com trastorno específico del lenguaje expresivo. Rev. CEFAC. 2005; 7(4): 419-25.

37. Capellini AS, Ferreira TL, Salgado CA, Ciasca SM. Desempenho de escolares bons leitores, com dislexia e com transtorno do déficit de atenção e hiperatividade em nomeação automática rápida. Rev. Soc. Bras. Fonoaudiol. 2007; 12(2): 114-9.

38. Zuanetti PA, Schneck APC, Manfredi AKS. Consciência fonológica e desempenho escolar. Rev. CEFAC. 2008; 10(2): 168-74.

39. Capellini AS, Padula NAMR, Santos LCA, Lourenceti MD, Carrenho EH, Ribeiro LA. Desempenho em consciência fonológica, memória operacional, leitura e escrita na dislexia familial. Pró-Fono Rev. Atual. Cient. 2007; 19(4): 374-80.

40. Rodrigues A, Befi-Lopes DM. Phonological working memory and its relationship with language development in children. Pró-Fono Rev de Atualização Científica. 2009, jan-mar; 21(1): 63-8.

http://dx.doi.org/10.1590/S1516-18462011005000099

RECEBIDO EM: 24/08/2010

ACEITO EM: 06/04/2011

Endereço para correspondência:

Sabrina Maria Pimentel da Cunha Pinto Tenório

Rua Dr. José Correia Filho, 308, Apt. 302

Edf. Trianon - Ponta Verde

Maceió - AL

CEP: $57035-130$

E-mail: sabrinampimentel@gmail.com 